failed, is not that it uses abstract models and traces interdependence between variables, but that it has sometimes been pseudo-planning, confined to a ceremonial superstructure, without being geared to where the action is.

The authors argue for the central importance of the annual budget. Though it is obviously true that no plan can be implemented unless it is integrated into the annual budget, there is a danger of mistaking a necessary, though minor, condition for the strategic one. Budgets are, at best, annual public expenditure plans. A focus on fiscal magnitudes, though essential for proper public accounting, obscures and evades the real activities. Links between fiscal (or even financial) expenditures and results are tenuous, especially in underdeveloped countries. There are no fixed coefficients between money expenditure and land reform, population policy, incomes policy, education, public health, nutrition. Foreign exchange budgeting, manpower budgeting, raw material budgeting are just as important as fiscal budgeting and even they do not exhaust the range of necessary policies. Proper public accounting is necessary to ensure, negatively, that public money is not spent extravagantly or corruptly, but it cannot ensure, positively, that it is spent according to social priorities and that the necessary complementary actions are taken. Budgeting is to planning what bookkeeping is to business management: without it, management is impossible; but with the best book-keeper in the world, a firm can go bankrupt. These points are not enough stressed by the authors, who see the plan essentially as a many-year public capital budget.

Much is made of the need for redundancy. In a piece of exquisite jargon, the authors write: "Broadly speaking, we can regard social poverty as a lack of functional redundancy" (Page 49). But there is a vast difference between reserves (which serve a purpose) and redundancies. A more analytical and quantitative approach would have made the distinction clear. It is now well known that in poor societies, not only unskilled labour, but also capital and technically trained professional manpower are redundant: but, alas, they are not reserves.

The authors make a large number of entirely fair and commonsensical criticisms of planning. "If we were asked to design a mechanism for decisions to maximise every known disability and minimise any possible advantage of poor countries, we could hardly do better than comprehensive, multi-sectoral planning" (Page 293). The need for unavailable information, for political stability, for consistent aims are cited as unattainable condi- tions. But perhaps the most serious criticism of planning is omitted, viz. that its very success, measured by coherence and consistency, becomes an obstacle to adaptation and innovation. Plans introduce an additional rigidity into societies already inflexible. Plans, for this reason, in spite of their declared intentions, are elements strengthening conservatism.

The conclusion is not, however, reliance on laissez-faire and the free play of market forces. The authors rightly point to the need for a combination of contingency planning, continuous budgeting and rolling planning, so that there can be adequate and speedy responses and adaptations to unforeseen events, both favourable and unfavourable.

The authors treat planners and planning as part of the social and political environment which they are supposed to plan. Planning the planners is not an invitation to an infinite regress but a reminder that there must be continuous mutual adaptation between plan objectives and social constraints.

Paul Streeten

\section{Details of sense organs}

The Ultrastructure of Sensory Organs. Edited by I. Friedmann. (NorthHolland: Amsterdam and London; American Elsevier: New York, 1974.) Dfl. $90 ; \$ 32.70$.

THIS collection of four essays is less general than the title implies; only vertebrates are described. Of their sense organs only taste buds (R. G. Murray), the organ of Corti $(\mathrm{H}$. Engström and H. W. Ades), the vertebrate retina (R. F. Dunn) and the olfactory mucosa (P. P. C. Graziadei) are included. The preface indicates the intention of the articles to summarise "the immense and rapid progress that has been made in electron microscopy" for "anatomists, physiologists, pathologists and clinicians". Only Engström and Ades make some attempt to meet the latter two classes of busy people part way. Anatomists are best treated in that all the articles provide descriptive anatomical accounts while remaining substantially innocent of physiological commentary or synthesis of the two approaches.

Thus the essays have to be judged not as summaries of the contributions ultrastructural studies are making to understanding of the mechanisms of action of the organs treated, but as summaries of anatomical information available up until about 1970-71. Most of the summaries are competent but, perhaps because of editorial direction, they all seem of rather even emphasis; so that exciting new developments and the relative importance, or accuracy, of different and differing observations are hard to discern. Perhaps the main point of the articles was to provide a review of the literature together with an atlas of ultrastructure. There are numerous electron micrographs including a fair number, especially of the retina, that have not previously been published. For the non-specialist in a particular sense organ the essays provide a reasonably readable guide to the literature of the past twenty years. There are useful summary tables of data, for example in the article on the retina. But for the readership suggested, and given that the articles are purely anatomical, a greater use of summary diagrams, perhaps at the expense of some of the electron micrographs, would have been more helpful to ready comprehension. The essays are probably most useful for someone beginning an interest in the structure of a particular sense organ. Most people are likely quickly to prefer the more detailed and often more synthetic articles of the currently appearing Handbook of Sensory $\begin{array}{ll}\text { Physiology. } & \text { B. B. Boycoutt }\end{array}$

\section{Computers in mathematics}

Computer Approaches to Mathematical Problems. By Jurg Nievergelt, J. Craig Farrar and Edward M. Reingold. Pp. xii +267. (Prentice-Hall, Englewood Cliffs, 1973.) $\$ 8.95$.

AfTer an introductory chapter on languages, including some remarks on the parenthesis notation and the polish notation, there is a chapter on combinatorial computing. This includes a discussion of block designs, latin squares. scheduling and tiled rectangles; and also an account of various graph algorithms, for shortest paths, spanning trees and sorting. There follows a chapter on the use of computers in game playing and decision making and another chapter on random numbers and their use in Monte Carlo methods and in simulation. Computing in number theory is next considered and there is a review of the position in the computing of $\sqrt{ } 2, e$ and $\pi$. For example, in 1967 the value of $\pi$ was computed to 500,000 decimal places. Finally there is a section on the use of computers in calculating large prime numbers. The largest known Mersenne prime, by 1971 , is $2^{p}-1$ where $p=19937$. The book closes with a chapter on logic and computers.

This book will be of particular interest to the mathematician interested in the use of computers in pure mathematics. But in this connection, it is a little surprising to find no reference to the use of computers in the theory of finite groups. It will also be relevant for students of operational research mathematics. L. S. GODDARD 